Pacific Journal of Mathematics

CODES, TRANSFORMS AND THE SPECTRUM OF THE 


\title{
CODES, TRANSFORMS AND THE SPECTRUM OF THE SYMMETRIC GROUP
}

\author{
Paul H. Edelman and Dennis White
}

Let $G_{n}$ be the graph of permutations with edges drawn between permutations differing by an adjacent transposition. Using the Kazhdan-Lusztig representations of $S_{n}$ and combinatorial arguments, we show that integers frequently occur in the spectrum of $G_{n}$. That 0 and -1 are among the integers which arise has application to finite Radon transforms and to existence of perfect 1 -codes on $G_{n}$.

1. Introduction. Let $G_{n}$ denote the graph with vertices labeled by the permutations of $\{1, \ldots, n\}$ and with edges drawn between two vertices if and only if the two permutations differ by an adjacent transposition. This graph is sometimes called the "permutohedron" [Be]. It is also the graph of the Hasse diagram for the weak order of the symmetric group [Bj]. We say that a number is an eigenvalue of $G_{n}$ if it is an eigenvalue of its adjacency matrix. Similarly we refer to the spectrum of $G_{n}$ when we really mean the spectrum of the adjacency matrix.

In this paper we investigate the occurrence of integer eigenvalues for this graph. We were originally led to this investigation because of a coding problem: does there exist a perfect 1-code on $G_{n}$ ? That is, is there a collection of vertices $C$ of $G_{n}$ such that the sets $P_{v}=$ $\{w \mid w=v$ or $w$ adjacent to $v\}$, for each $v \in C$, partition all the vertices of $G_{n}$ ? Such a code exists for $n=3$ (see Figure 1), but not for any other $n<12$. We will show that if such a code exists, then -1 must be an eigenvalue of $G_{n}$. We will also show that -1 is always an eigenvalue of $G_{n}$.

Questions about the spectrum of $G_{n}$ also arise in other settings. In a later section we will define a finite Radon transform on $S_{n}$ whose invertibility is dependent on the existence (or non-existence) of certain eigenvalues of $G_{n}$. The spectrum of $G_{n}$ is also relevant in analyzing the behavior of certain shuffling problems [DS]. While most of these applications involve knowing only whether 0 or 1 is in the spectrum, the more general problem of what integers appear in the spectrum leads to better techniques in resolving these specific cases and is a more 
appropriate level of generality. In fact the integer spectrum seems to be very structured and we make a number of conjectures concerning its behavior in the final section.

Our techniques are combinatorial and algebraic. We will search for integer eigenvalues in the irreducible subspaces corresponding to hook shapes. Surprisingly, this seems to be where these eigenvalues occur most frequently. We will use the Kazhdan-Lusztig representations (see [KL]) for $S_{n}$ to restate our eigenvalue problem in terms of the Hasse diagram of Young's lattice on a rectangle.

This paper is organized as follows: In the next section we discuss the coding problem that motivates this research and show how it relates to the spectrum of $G_{n}$. Section 3 will discuss some elementary facts about this spectrum. In $\S 4$ we assemble the relevant results of the Kazhdan-Lusztig theory of representations, which we then apply in $\S 5$. Section 6 contains an explicit computation of a class of eigenvectors for $G_{n}$. The eigenvalues we produce have a particularly nice form and allow us to construct types of codes for $G_{n}$, although they are not perfect 1-codes. This is followed by an application of our results to the invertibility of a Radon transform on $S_{n}$, and, finally, in $\S 8$ we make a number of conjectures concerning the spectrum of $G_{n}$.

We close this section by setting some of our notation. For $n$ and $k$ positive integers, $1<k<n$, let $[n]=\{1,2, \ldots, n\},[k, n]=$ [n] $-[k-1]$. If $\sigma \in S_{n}$ then we will let $\sigma_{i}=\sigma(i)$. If $A \subseteq[n]$ let $\left.\sigma\right|_{A}$ be the ordering on $A$ induced by $\sigma$, i.e.,

$$
\left.\sigma\right|_{A}=\sigma_{i_{1}} \sigma_{i_{2}} \cdots \sigma_{i_{h}} \quad \text { where } i_{1}<i_{2}<\cdots<i_{k} \text { and }\left\{\sigma\left(i_{j}\right)\right\}=A
$$

For $1 \leq i \leq n-1$ we reserve $\tau^{i}$ for the adjacent transposition $(i, i+$ 1) $\in S_{n}$. Our multiplication in $S_{n}$ will be from right to left.

If $G$ is a graph with two adjacent vertices $v$ and $w$ then we say that $v \sim w$. Let $A(G)$ be the (vertex) adjacency matrix of $G$, i.e., the matrix whose rows and columns are indexed by the vertices of $G$, with an entry 1 if two vertices are adjacent and 0 otherwise. Entries on the diagonal are always 0 . As noted before, we will abuse terminology somewhat and say that $G$ has certain matrix properties when we really mean that its adjacency matrix does. If $v \in G$, the closed neighborhood of $v$ is the set of vertices adjacent to $v$ with $v$ itself.

2. Codes. We will let $S_{n}$ denote the elements of the symmetric 


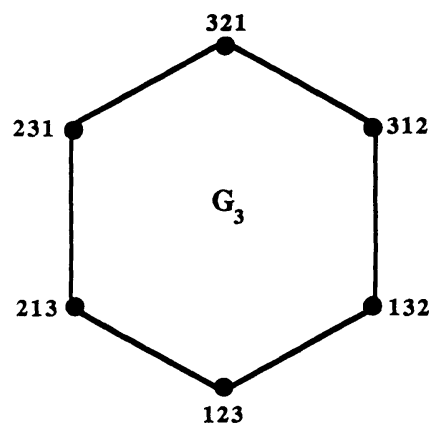

FIGURE 1

group. If $A \subset S_{n}$, denote by

$$
\widehat{A}=\sum_{\sigma \in A} \sigma \in \mathbb{C} S_{n},
$$

the corresponding element of the group algebra of $S_{n}$. Let

$$
T=\{(t, t+1) \mid 1 \leq t \leq n-1\}
$$

and

$$
T_{1}=T \cup \mathrm{id} .
$$

We may now state the coding problem: Does there exist a subset

$$
C \subset S_{n}
$$

such that

$$
\widehat{C} \widehat{T}_{1}=\widehat{S}_{n} ?
$$

If such a $C$ exists, we say a code exists for $S_{n}$. For example, if $n=3$, then

$$
C=\left\{\left(\begin{array}{lll}
1 & 2 & 3 \\
1 & 2 & 3
\end{array}\right),\left(\begin{array}{lll}
1 & 2 & 3 \\
3 & 2 & 1
\end{array}\right)\right\}
$$

This is a variation of the coding problem of Rothaus and Thompson [RT] who replace $T_{1}$ with $T_{1}^{\prime}$ with

$$
T_{1}^{\prime}=\{(i, j) \mid 1 \leq i<j \leq n\} \cup \mathrm{id} .
$$

Working with $T_{1}^{\prime}$ has certain technical advantages, since $T_{1}^{\prime}$ is a conjugacy class and hence $\widehat{T}_{1}^{\prime}$ lies in the center of $\mathbb{C} S_{n}$. This allows the use of character theory to analyze the behavior of $\widehat{T}_{1}^{\prime}$. We know of no way to apply character theory in a similar way to our problem.

Let $G_{n}$ be the graph described in $\S 1$. That is, the vertex set of $G_{n}$ is $S_{n}$ and two permutations are adjacent if they differ by an adjacent transposition ( $G_{3}$ is pictured in Figure 1 ). Then the adjacency matrix 
of $G_{n}$ is the same as the image of $T$ under the right-regular representation of $S_{n}$. A code for $S_{n}$ will correspond to a set of vertices $C \subset S_{n}$ such that the closed neighborhoods of $C$ exactly partition the vertices of $G_{n}$. Such a partitioning is sometimes called a perfect 1-code.

Now suppose $\chi$ is a representation of $S_{n}$. Let $A^{\chi}=\chi(\widehat{A})$.

Suppose $G \subset S_{n}$ is a subgroup of $S_{n}$. Then the natural action of $S_{n}$ on the cosets $S_{n} / G$ gives rise to a permutation representation of $S_{n}$ which we call $\chi_{G}$.

Proposition 2.1. Let $G$ be a subgroup of $S_{n}$ and $\chi_{G}$ the permutation representation of $S_{n}$ on the cosets $S_{n} / G$. If the equation

$$
M T_{1}^{\chi_{G}}=S_{n}^{\chi_{G}}
$$

has no integral solution matrix $M$, then no code exists.

Proof. Suppose a code $C$ exists. Then projecting into the representation $\chi_{G}$ we get

$$
C^{\chi_{G}} T_{1}^{\chi_{G}}=S_{n}^{\chi_{G}} \text {. }
$$

But $C$ is a subset of $S_{n}$ and therefore $C^{\chi_{G}}$ is integral.

The following theorem generalizes an observation of Stanley [St].

THEOREM 2.2. Let $G$ be a subgroup of $S_{n}$ and $\chi_{G}$ the permutation representation of $S_{n}$ on cosets $S_{n} / G$. If $T_{1}^{\chi_{G}}$ is invertible and $n \nmid|G|$, then no code exists.

Proof. Suppose a code $C$ exists. By Proposition 2.1, the unique solution matrix

$$
S_{n}^{\chi_{G i}} T_{1}^{\chi_{G}-1}
$$

must be integral. But all of the entries of this matrix are $|G| / n$.

Theorem 2.2 has some important corollaries.

Corollary 2.3. If $T_{1}$ is invertible then no code exists for $S_{n}$.

Proof. Let $G=\{\mathrm{id}\}$. Then $\chi_{G}$ is exactly the right-regular representation of $S_{n}$ and $T_{1}^{\chi_{G}}=T_{1}$. 
COROllary 2.4. No code exists for $S_{n}$ if $n+(n-1)$ ! and $n>3$.

Proof. Let $G=S_{n-1} \times S_{1}$. Then

$$
T_{1}^{\chi_{G}}=\left(\begin{array}{ccccccc}
n-1 & 1 & 0 & & & \ldots & 0 \\
1 & n-2 & 1 & 0 & & \ldots & 0 \\
0 & 1 & n-2 & 1 & 0 & \ldots & 0 \\
\vdots & & & & \ddots & & \vdots \\
0 & & \ldots & 0 & 1 & & n-1
\end{array}\right)
$$

which is invertible.

Corollary 2.5. No code exists for $S_{n}$ if $n=4$ or $n$ is prime $\geq 5$.

Proof. This follows from Corollary 2.4.

THEOREM 2.6. No code exists for $S_{n}$ if $3<n<12$.

Proof. Corollary 2.5 eliminates $n=4,5,7,11$. We have used a computer to verify the hypothesis of Theorem 2.2 for $n=8$ ( $G=$ $\left.S_{3} \times S_{3} \times S_{1} \times S_{1}\right)$, for $n=9 \quad\left(G=S_{5} \times S_{2} \times S_{2}\right)$, and for $n=10$ $\left(G=S_{4} \times S_{4} \times S_{2}\right)$. Finally, although we did not find a subgroup $G$ which satisfied the hypothesis of Theorem 2.2 for $n=6$, the subgroup $S_{2} \times S_{2} \times S_{2}$ does satisfy the hypothesis of Proposition 2.1.

Note that we did not apply Corollary 2.3 to any $n$. The reason is that the hypothesis of this corollary is never satisfied. We shall prove in $\S 5$ (Corollary 5.5) the following theorem.

THEOREM 2.7. $T_{1}$ is singular for $n \geq 2$.

The difficult part of applying Theorem 2.2 is knowing when $T_{1}^{\chi_{G}}$ is invertible. As noted in the proof of Theorem 2.6, even if this transformation is not invertible, that does not guarantee the existence of the code. For $n=6$, we checked all the Young subgroups which satisfied the non-divisibility condition, and we checked a number of other obvious subgroups (e.g., the 2- and 3-Sylow subgroups). In every case, the projection of $T_{1}$ was singular. In spite of this, no code exists for $n=6$.

Conjecture 2.8. No code exists for $S_{n}$ for all $n>3$.

3. The spectrum of $S_{n}$. Recall our observation that the adjacency matrix of $G_{n}$ is the action of the right-regular representation of $S_{n}$ on $T$. The following theorem follows immediately: 


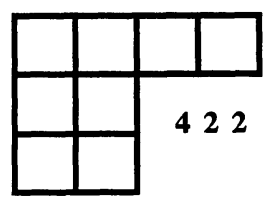

FIGURE 2

Proposition 3.1. $T$ is singular if and only if 0 is an eigenvalue of $G_{n}$ and $T_{1}$ is singular if and only if -1 is an eigenvalue of $G_{n}$.

This motivates us to study the spectrum of $G_{n}$ in general, and to seek other integer eigenvalues in particular. Let

$$
\operatorname{Spec}(T)=\{\text { integer eigenvalues of } \widehat{T}\} \text {. }
$$

Since $G_{n}$ is regular of degree $n-1$ and is bipartite, we have

Proposition 3.2. If $j \in \operatorname{Spec}(T)$, then $-j \in \operatorname{Spec}(T)$. Also, $n-1$, $-(n-1) \in \operatorname{Spec}(T)$ and if $j \in \operatorname{Spec}(T)$, then $|j| \leq n-1$.

It is well known that the irreducible representations of $S_{n}$ are indexed by integer partitions [JK]. Integer partitions are sometimes referred to as shapes, particularly when viewed as Ferrers diagrams, that is arrangements of rows of cells, left-justified and non-increasing in length. For example, Figure 2 gives the Ferrers diagram for the partition $422=42^{2}$. The conjugate partition of the partition $\lambda$, denoted $\lambda^{\prime}$, is obtained by transposing the Ferrers diagram. For example, $42^{2^{\prime}}=3^{2} 1^{2}$. A hook shape or hook partition is a partition of the form $m 1^{k}$. Two degenerate hook shapes are $(n)$ and $1^{n}$. We shall use $\lambda$ to denote either the shape or the corresponding irreducible representation of $S_{n}$.

For each irreducible representation $\lambda$ of $S_{n}$, let

$$
\operatorname{Spec}\left(T^{\lambda}\right)=\left\{\text { integer eigenvalues of } T^{\lambda}\right\} \text {. }
$$

We have

Proposition 3.3. If $j \in \operatorname{Spec}\left(T^{\lambda}\right)$, then $-j \in \operatorname{Spec}\left(T^{\lambda^{\prime}}\right)$, where $\lambda^{\prime}$ is the conjugate partition of $\lambda$. Also, $n-1 \in \operatorname{Spec}\left(T^{(n)}\right)$ and $-(n-1) \in$ $\operatorname{Spec}\left(T^{1^{n}}\right)$.

Proof. This follows from a result of Babai [Ba, Theorem 3.1].

In the following sections, we shall find that $\operatorname{Spec}\left(T^{\lambda}\right)$ is frequently non-empty when $\lambda$ is a hook shape. Also, for hook shapes it is quite 
easy to write down a combinatorial description of the entries of $T^{\lambda}$ using either Young's natural representation [GM] or the KazhdanLusztig representations [GM]. We shall use the Kazhdan-Lusztig representations, although many of the theorems we state could also be proved using Young's natural representation.

4. The Kazhdan-Lusztig representations. Let $A=[n-k]$ and $B=$ $[n-k+1, n]$ and consider the set of all permutations $\sigma \in S_{n}$ with the properties that

(1) $\left.\sigma\right|_{A}=12 \cdots n-k$,

(2) $\left.\sigma\right|_{B}=n n-1 \cdots n-k+1$,

(3) $\sigma(n)=n-k$. Call this set $P_{k, n-k}$. Equivalently $P_{k, n-k}$ is the set of shuffles of the permutations $\alpha=12 \cdots n-k$ and $\beta=$ $n n-1 \cdots n-k+1$ with the property that $n-k$ appears last. It is clear that $\left|P_{k, n-k}\right|=\left(\begin{array}{c}n-1 \\ k\end{array}\right)$. Another less obvious definition of $P_{k, n-k}$ is the set of permutations $\sigma \in S_{n}$ such that under the Schensted correspondence [Kn, Section 5.1.4]

$$
P(\sigma)=\begin{array}{cc}
1 \\
n-k+1
\end{array} \quad \text { } 2 \cdots n-k
$$

Partially order $P_{k, n-k}$ by using the induced order from the weak order of $S_{n}$, i.e., say that $\sigma \leq \tau$ if $\tau$ can be gotten from $\sigma$ by a sequence of adjacent transpositions each moving the larger number to the left. We may think of $P_{k, n-k}$ as a graph by using the cover relations of this order to be the edges. Thus we say that $\sigma \sim \tau$ if $\sigma$ differs from $\tau$ by an adjacent transposition.

Associated with each $\sigma \in P_{k, n-k}$ there is a set

$$
D(\sigma)=\left\{\tau^{i} \mid \sigma \tau^{i} \leq \sigma\right\}
$$

where $\tau^{i}=(i, i+1)$. The Kazhdan-Lusztig theory of representations allows us to use the graph $P_{k, n-k}$ and the sets $\{D(\sigma)\}$ to produce an irreducible representation of $S_{n}$ associated with the shape $n-k, 1^{k}$ as follows:

For each $\tau^{i}, 1 \leq i \leq n-1$, define $M_{i}$ to be transformation on the formal vector space spanned by $\left\{\sigma \in P_{k, n-k}\right\}$ where

$$
M_{i}(\sigma)= \begin{cases}-\sigma & \text { if } \tau^{i} \in D(\sigma), \\ \sigma+\sum_{\substack{\sigma^{\prime} \sim \sigma \\ \tau^{\prime} \in D\left(\sigma^{\prime}\right)}} \sigma^{\prime} & \text { if } \tau^{i} \notin D(\sigma) .\end{cases}
$$


The next theorem follows from the work of Kazhdan and Lusztig [KL]. For an exposition of this material from a combinatorial perspective see [GM].

THEOREM 4.1 [KL, Theorem 1.4] [GM, Fact 14]. The set of matrices $\left\{M_{i} \mid 1 \leq i \leq n-1\right\}$ are representing matrices for the adjacent transpositions of $S_{n}$ in the irreducible representation corresponding to the shape $n-k, 1^{k}$.

Before proceeding to the main theorem in this section we require two lemmas:

Lemma 4.2. For $\sigma \in P_{k, n-k}, \tau^{i} \in D(\sigma)$ if and only if

$$
\sigma_{i} \in[n-k+1, n] \text {. }
$$

Proof. This follows immediately from the fact that $\sigma \in P_{k, n-k}$ means that $\sigma$ is the shuffle of the two permutations $\alpha$ and $\beta$.

LEMMA 4.3. Let $\sigma, \tau \in P_{k, n-k}$. If $\sigma \tau^{j}=\tau$ then either $\left\{\tau^{j}\right\}=$ $D(\sigma)-D(\tau)$ or $\left\{\tau^{j+1}\right\}=D(\sigma)-D(\tau)$.

Proof. From Lemma 4.2 it follows that if $\sigma, \tau \in P_{k, n-k}$ and $\sigma \sim \tau$ then $D(\sigma)-D(\tau)$ can have cardinality at most 1. If $\sigma<\tau$ and $\sigma \tau^{j}=\tau$ then $\sigma_{j} \in[n-k]$ and $\sigma_{j+1} \in[n-k+1, n]$ and hence by Lemma $4.2\left\{\tau^{j+1}\right\}=D(\sigma)-D(\tau)$. Similarly if $\tau<\sigma$ and $\sigma \tau^{j}=\tau$ then $\tau^{j} \in D(\sigma)$ and hence $\sigma_{j} \in[n-k+1, n]$ and $\sigma_{j+1} \in[n-k]$. Thus $\tau^{j} \notin D(\tau)$ and the lemma is complete.

THEOREM 4.4 [St]. For $\lambda=n-k, 1^{k}, T^{\lambda}$ can be represented by the matrix $A+(n-2 k-1) I$ where $A$ is the adjacency matrix of $P_{k, n-k}$.

Proof. By Theorem 4.1 we have that $T=\sum_{i=1}^{n-1} M_{i}$. Then, for $\tau \neq \sigma$, the entry

$$
(T)_{\tau, \sigma}=\left|\left\{j \in[n-1] \mid \tau^{\jmath} \in D(\sigma)-D(\tau), \sigma \sim \tau\right\}\right|
$$

From Lemma 4.3 it is clear that $(T)_{\tau, \sigma}$ is 1 or 0 . If $\sigma \not \tau$ then $(T)_{\tau, \sigma}=0$. If $\sigma \sim \tau$ then from Lemma 4.3 we have that $(T)_{\tau, \sigma}=1$. From Lemma 4.2 we conclude that $(T)_{\sigma \sigma}=n-2 k-1$. This finishes the proof. 


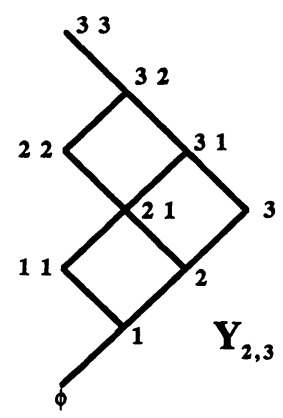

Figure 3

There is another way to think of the poset $P_{k, n-k}$ which will be very useful in what follows. For $\sigma \in P_{k, n-k}$ let $\lambda(\sigma)$ be the partition

$$
\lambda(\dot{\sigma})=\left\{2 n-k-t-\sigma^{-1}(t) \mid n \geq t \geq n-k+1\right\} .
$$

It is easy to check that this map is an order-preserving bijection between $P_{k, n-k}$ and the set of partitions which fit into a $k \times(n-k-1)$ rectangle ordered by containment. This latter poset is called Young's lattice [SW, Section 2.1] on a $k \times(n-k-1)$ rectangle and will be denoted $Y_{k, n-k-1}$. We will see in subsequent sections the advantages of working with partitions instead of permutations. The Hasse diagram of $Y_{2,3}$ is shown in Figure 3.

Corollary 4.5. If $j \in \operatorname{Spec}\left(T^{\lambda}\right), \lambda=n-k, 1^{k}$, then $2(n-2 k-1)$ $-j \in \operatorname{Spec}\left(T^{\lambda}\right)$.

Proof. This follows from Theorem 4.4 and the fact that $Y_{k, n-k-1}$ is bipartite.

5. The eigenvalues of Young's lattice. As we have shown in the previous section, information regarding the eigenvalues of $S_{n}$ can be obtained from the eigenvalues of Young's lattice on a rectangle. We exploit this connection in this section.

THEOREM 5.1. If $\lambda=n-1,1$, then $n-2, n-4 \in \operatorname{Spec}\left(T^{\lambda}\right)$ if $n \equiv 0 \bmod 3$ and $n-3 \in \operatorname{Spec}\left(T^{\lambda}\right)$ if $n \equiv 0 \bmod 2$. Furthermore, these are the only members of $\operatorname{Spec}\left(T^{\lambda}\right)$.

Proof. This result can be gotten directly from the matrix in the proof of Corollary 2.4. That representation will have one copy of $\lambda$ and one copy of the irreducible representation $(n)$, which will contribute the eigenvalue $n-1$. 
However, this theorem also follows easily from Theorem 4.4. Since $Y_{1, n-2}$ is a chain, $A$ is tridiagonal with 0 's on the main diagonal and 1 's along the super- and sub-diagonals. The characteristic polynomial of this matrix is a Chebyschev polynomial (see [CDS, Section 2.6]). The eigenvalues are well known to be $2 \cos k \pi / n, k=1, \ldots, n-1$.

Corollary 5.2. If $\lambda=2,1^{n-2}$, then $-(n-2),-(n-4) \in$ $\operatorname{Spec}\left(T^{\lambda}\right)$ if $n \equiv 0 \bmod 3$ and $-(n-3) \in \operatorname{Spec}\left(T^{\lambda}\right)$ if $n \equiv 0 \bmod 2$. Furthermore, these are the only members of $\operatorname{Spec}\left(T^{\lambda}\right)$.

\section{THEOREM 5.3. If $\lambda=\lfloor n / 2\rfloor+1,1^{\lfloor(n-1) / 2\rfloor}$ then $+1 \in \operatorname{Spec}\left(T^{\lambda}\right)$.}

Proof. We will prove this theorem by explicitly producing an eigenvector for the adjacency matrix of $Y_{\lfloor n / 2\rfloor,\lfloor(n-1) / 2\rfloor}$ with the appropriate eigenvalue. The proof then follows from Theorem 4.4. Consider first the case where $n$ is odd. We are then considering the graph $Y_{(n-1) / 2,(n-1) / 2}$.

Let $\mathbf{v}$ be the vector in the formal vector space spanned by the elements of $Y_{(n-1) / 2,(n-1) / 2}$ whose coefficient in the $\lambda$ coordinate is given by

$$
\mathbf{v}(\lambda)= \begin{cases}0 & \text { if } \lambda \neq \lambda^{\prime}, \\ -1^{(|\lambda|-d(\lambda)) / 2} & \text { if } \lambda=\lambda^{\prime},\end{cases}
$$

where $|\lambda|$ is the number of cells in $\lambda, d(\lambda)$ is the size of the Durfee square of $\lambda$ (the number of cells along the main diagonal), and $\lambda^{\prime}$ is the conjugate of $\lambda$. We leave it as an exercise for the reader to check that this is an eigenvector of eigenvalue 1 .

Now suppose $n$ is even. Then we consider the graph of $Y_{(n / 2)-1, n / 2}$ and we must find an eigenvector with eigenvalue 0 for its adjacency matrix. Define a partition to be quasi-self-conjugate (qsc) if its $(d+1)$ st column has size $d$ and its removal results in a selfconjugate partition, where $d=d(\lambda)$. Define a vector $\mathbf{v}$ by

$$
\mathbf{v}(\lambda)= \begin{cases}0 & \text { if } \lambda \text { is not qsc } \\ -1^{|\lambda| / 2} & \text { if } \lambda \text { is qsc. }\end{cases}
$$

Again we leave it as an exercise to check that this is an eigenvector for $A_{(n / 2)-1, n / 2}$ with eigenvalue 0 .

Corollary 5.4. If $\lambda=\lfloor(n-1) / 2\rfloor+1,1^{\lfloor n / 2\rfloor}$, then $-1 \in \operatorname{Spec}\left(T^{\lambda}\right)$.

Corollary 5.5 (Theorem 2.7). $T_{1}$ is singular for $n \geq 2$.

Proof. This is immediate from Corollary 5.4 and Proposition 3.1. 


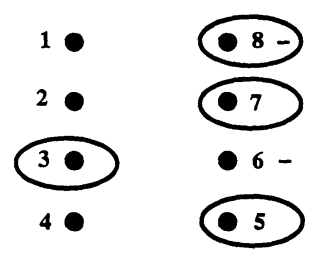

FIGURE 4

At this point we give an alternative proof of Theorem 5.3, which lends itself to generalization in different ways than our previous one (see for instance $\S 6$ ).

Let $W_{a, b}$ be the set of words of length $a+b$ which use only the alphabet $\{0,1\}$, with exactly $a 1$ 's. Then it is clear that the graph $Y_{a, b}$ is isomorphic to the graph on $W_{a, b}$ where two words are adjacent if they differ by a transposition of an adjacent 0 and 1 . For this next proof, we will think of $Y_{a, b}$ as this graph on $W_{a, b}$.

Alternative proof for Theorem 5.3. Consider first the case where $n$ is odd. Then $a=b=(n-1) / 2$. Let $l=(n+1) / 2$ and

$$
E=\left\{u \in W_{a, b} \mid u_{i}+u_{a+b-i}=1,1 \leq i<l\right\} .
$$

Partition $E$ into

$$
E^{+}=\left\{u \in E \mid u_{l+2}+u_{l+4}+\cdots \text { is even }\right\}
$$

and

$$
E^{-}=\left\{u \in E \mid u_{l+2}+u_{l+4}+\cdots \text { is odd }\right\} .
$$

Figure 4 gives an example of an element of $E^{-}$for $n=9$. Now define the vector $\mathbf{v}$ so that

$$
\mathbf{v}_{u}= \begin{cases}+1 & \text { if } u \in E^{+} \\ -1 & \text { if } u \in E^{-} \\ 0 & \text { otherwise }\end{cases}
$$

If $u \notin E$ is adjacent to an element of $E$, then $u$ is adjacent to exactly one element of $E^{+}$and one element of $E^{-}$. If $u \in E$, then $u$ is adjacent to exactly one other $u^{\prime} \in E$ and $u \in E^{+}$if and only if $u^{\prime} \in E^{-}$.

Thus $\mathbf{v}$ is an eigenvector corresponding to the eigenvalue +1 for the graph $W_{a, b}$. Now suppose $n$ is even. Then $b=a+1=n / 2$. Let $l=n / 2$ and

$$
E=\left\{u \in W_{a, b} \mid u_{i}+u_{a+b-i}=1,1 \leq i<l \text { and } u_{l}=1\right\} .
$$




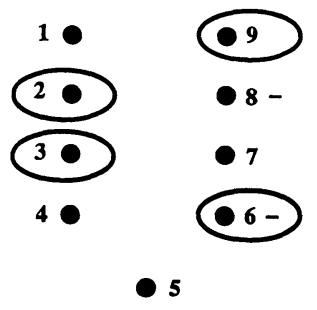

FIGURE 5

Partition $E$ into

$$
E^{+}=\left\{u \in E \mid u_{l+1}+u_{l+3}+\cdots \text { is even }\right\}
$$

and

$$
E^{-}=\left\{u \in E \mid u_{l+1}+u_{l+3}+\cdots \text { is odd }\right\} .
$$

Figure 5 gives an example of an element of $E^{-}$for $n=10$. Define the vector $\mathbf{v}$ as before.

Again, if $u \notin E$ is adjacent to an element of $E$, then $u$ is adjacent to exactly one element of $E^{+}$and one element of $E^{-}$. If $u \in E$, then $u$ is not adjacent to any other element of $E$.

Thus $\mathbf{v}$ is an eigenvector corresponding to the eigenvalue 0 for the graph $W_{a, b}$.

THEOREM 5.5. If $\lambda=n-k, 1^{k}$ and $n$ is even, then $n-1-2 k \in$ $\operatorname{Spec}\left(T^{\lambda}\right)$ for $k=1, \ldots, n-2$. If $n$ is odd, then $n-1-2 k \in \operatorname{Spec}\left(T^{\lambda}\right)$ for $k=2,4, \ldots, n-2$.

Proof. Consider $Y_{k, n-k-1}$. It is a bipartite graph, so we can partition it into two disjoint independent sets, $Y_{k, n-k-1}=U \cup V$. Furthermore,

$$
|| U|-| V||=\left[\begin{array}{c}
n-1 \\
k
\end{array}\right]_{-1} .
$$

But is is easy to see that

$$
\left[\begin{array}{c}
n-1 \\
k
\end{array}\right]_{-1}=0
$$

if and only if $k$ and $n-k-1$ are both odd.

Therefore, if $n$ is even or if $n$ is odd and $k$ is even, the graph $Y_{k, n-k-1}$ will be unbalanced bipartite. The adjacency matrix of such a graph is singular [CDS, Theorem 8.2].

We conclude this section with one final theorem. 


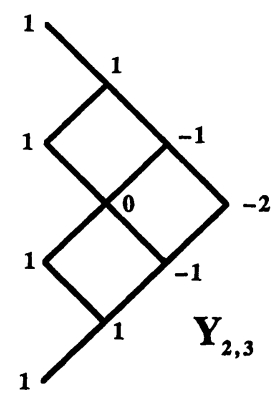

FigURE 6

THEOREM 5.6. If $\lambda=n-2,1^{2}$, then $n-4, n-6 \in \operatorname{Spec}\left(T^{\lambda}\right)$ for $n \equiv 0 \bmod 5$ and for $n \equiv 0 \bmod 6$.

Proof. We consider first $n \equiv 0$ mod 6 . Figure 6 gives an eigenvector for the graph $Y_{2,3}$ for the eigenvalue 1 . We will call this eigenvector a tile. This particular eigenvector is called a positive tile. The negative of this eigenvector will be called a negative tile. We will call the values assigned to a vertex by the eigenvector the vertex value.

To indicate the general picture, we show the eigenvector for the graph $Y_{2,15}$ for eigenvalue 1 in Figure 7. We have marked off regions of the graph which are separated by vertices with value 0 . Each of these regions is a positive or negative tile. Since each tile is an eigenvector for eigenvalue 1 , within a tile the eigenvector condition is maintained. It is easy to verify that each tile can be made positive or negative in a manner such that negative tiles share borders only with positive tiles and vice versa. We call such a tiling a consistent tiling. It is also easy to check that for a consistent tiling, the sum of vertex values about a boundary vertex must be zero. In Figures 8 and 9 we indicate graphically the tiling of $Y_{2,33}$ and $Y_{2,39}$.

For $n \equiv 0 \bmod 5$ we use the eigenvector of the graph $Y_{2,2}$ for eigenvalue 1 given in Figure 10 as our positive tile. Then the argument proceeds as above.

The $n-6$ case follows from Corollary 4.5.

6. Eigenvectors for $G_{n}$. In the previous section we proved the existence of certain eigenvalues for $T$ by projecting it into an irreducible representation and computing an eigenvector within that representation. In this section we will work with the adjacency matrix of $G_{n}$, i.e., with the projection of $T$ into the right-regular representation and produce a class of eigenvectors. The eigenvalues of the eigenvectors we produce are known to be in the spectrum of $G_{n}$ by Theorem 5.5. 


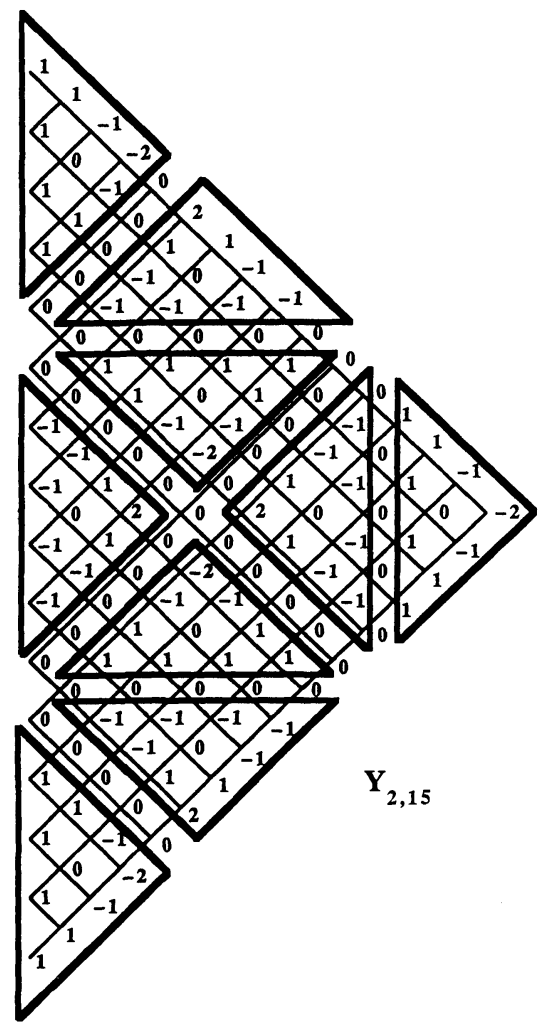

FIGURE 7

However, we do not know that the eigenvectors we construct do in fact project solely into the representation indicated by Theorem 5.5. The eigenvectors we produce also have a particularly nice structure which allows us to prove some interesting corollaries.

Suppose $\pi \in S_{n}$ and $1 \leq k \leq n$ is an odd number. Let $\mathbf{w} \in W_{k, n-k}$ be the word defined by

$$
\mathbf{w}_{i}= \begin{cases}1 & \text { if } \pi_{i} \in\{1, \ldots, k\}, \\ 0 & \text { if } \pi_{i} \in\{k+1, \ldots, n\} .\end{cases}
$$

If $\mathbf{w}_{j}=0$, define

$$
\eta_{j}=\left|\left\{i<j \mid \mathbf{w}_{i}=1\right\}\right| \text {. }
$$

Let

$$
\operatorname{sgn}(\mathbf{w})=\prod_{j: \mathbf{w},=0}(-1)^{\lfloor\eta, / 2\rfloor} .
$$

Finally, let $\hat{\pi}=\left.\pi\right|_{\{1, \ldots, k\}}$ and define

$$
\alpha(\pi)=\operatorname{sgn}(\mathbf{w}) \operatorname{sgn}(\hat{\pi}) .
$$




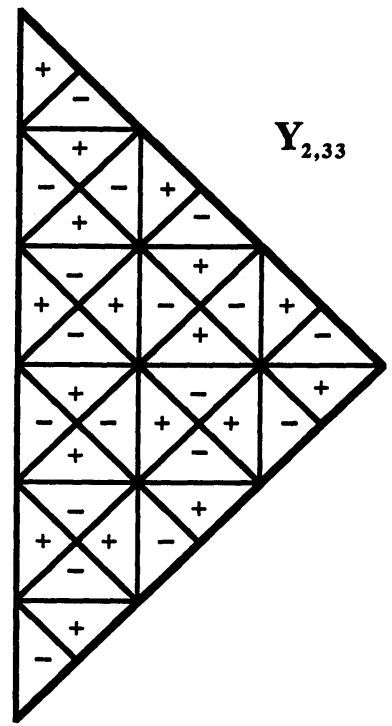

FIGURE 8

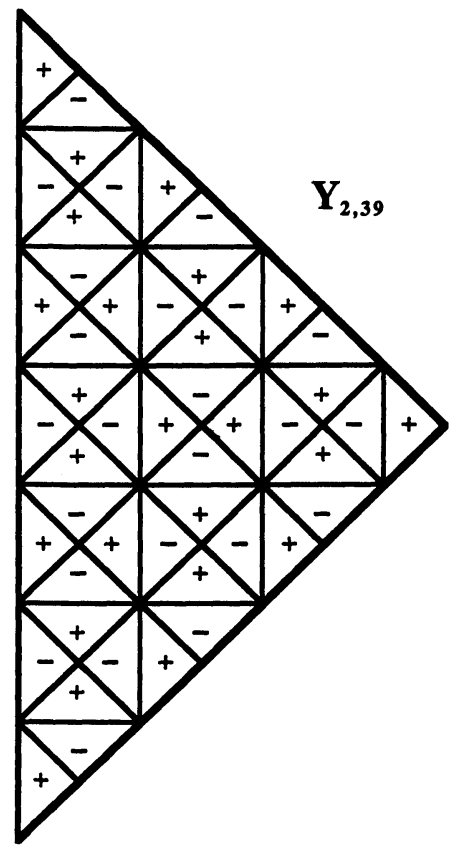

FIGURE 9

THEOREM 6.1. $\alpha$ is an eigenvector corresponding to the eigenvalue $n-2 k+1$.

Note that this is an explicit construction of the eigenvectors for 


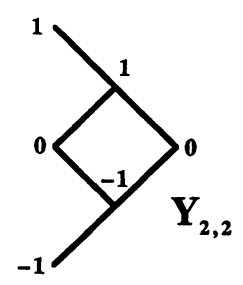

\section{FIGURE 10}

eigenvalues $n-4 l-1$ for $l=0,1, \ldots,(n-1) / 2$ for $n$ odd and for the eigenvalues $n-2 l-1$ for $l=0,1, \ldots, n-1$ for $n$ even.

Proof. Let $\operatorname{Adj}(\pi)$ denote the elements of $S_{n}$ which are adjacent to $\pi$ in $G_{n}$. Write $\pi^{i} \in \operatorname{Adj}(\pi)$ to denote the permutation gotten from $\pi$ by switching $\pi_{i}$ and $\pi_{i+1}$. Write $\mathbf{w}^{i}$ to denote the word corresponding to $\pi^{i}$. We will show

$$
\sum_{\pi^{\prime} \in \operatorname{Adj}(\pi)} \alpha\left(\pi^{i}\right)=(n-2 k+1) \alpha(\pi) .
$$

First, partition $\operatorname{Adj}(\pi)$ into

$$
U(\pi)=\left\{\pi^{i} \in \operatorname{Adj}(\pi) \mid \mathbf{w}^{i}=\mathbf{w}\right\}
$$

and

$$
V(\pi)=\operatorname{Adj}(\pi)-U(\pi) .
$$

Note that for $\pi^{i} \in U(\pi), \operatorname{sgn}\left(\mathbf{w}^{i}\right)=\operatorname{sgn}(\mathbf{w})$, but $\operatorname{sgn}(\hat{\pi})$ and $\operatorname{sgn}\left(\hat{\pi}^{i}\right)$ may differ; while for $\pi^{i} \in V(\pi), \operatorname{sgn}\left(\hat{\pi}^{i}\right)=\operatorname{sgn}(\hat{\pi})$, but $\operatorname{sgn}\left(\mathbf{w}^{i}\right)$ and $\operatorname{sgn}(\mathbf{w})$ may differ.

Let

$$
\text { Flat }_{q}(\mathbf{w})=\left|\left\{i \mid \mathbf{w}_{i}=\mathbf{w}_{i+1}=q\right\}\right|,
$$

for $q=0,1$. That is $\operatorname{Flat}_{q}(\mathbf{w})$ is the number of adjacent pairs of $q$ 's in $\mathbf{w}$. Let

$$
\operatorname{FlatDif}(\mathbf{w})=\text { Flat }_{0}(\mathbf{w})-\text { Flat }_{1}(\mathbf{w}) \text {. }
$$

We rewrite the left-hand side of (1) as

$$
\sum_{\pi^{i} \in U(\pi)} \alpha\left(\pi^{i}\right)+\sum_{\pi^{i} \in V(\pi)} \alpha\left(\pi^{i}\right) .
$$

The first sum is

$$
\sum_{\pi^{i} \in U(\pi)} \alpha\left(\pi^{i}\right)=\alpha(\pi) \text { FlatDif }(\mathbf{w})
$$


Suppose $\mathbf{w}$ has a subword of 0 's with no intervening 1 . That is, suppose $i \geq 1$ and $j<n$ are such that $\mathbf{w}_{i+1}=\mathbf{w}_{i+2}=\cdots=\mathbf{w}_{j}=0$ and $\mathbf{w}_{i}=\mathbf{w}_{j+1}=1$. It is easy to see from the definition of $\operatorname{sgn}(\mathbf{w})$ that $\operatorname{sgn}\left(\mathbf{w}^{i}\right)=-\operatorname{sgn}\left(\mathbf{w}^{j}\right)$.

Therefore in the second sum of (2) almost all terms will pair off and cancel. We are left only with a (perhaps empty) leading string of 0 's and a (perhaps empty) trailing string of 0 's. Let us write $T_{2}$ for this second sum. We now consider four cases.

Case 1. w has no leading or trailing string of 0 's. Clearly, then $T_{2}=0$. Also, it is easy to see that FlatDif $=n-2 k+1$.

Case 2. w has a leading string of 0 's but no trailing string of 0 's. Let $s$ be the location of the last 0 in the leading string. Then $\operatorname{sgn}\left(\mathbf{w}^{s}\right)=\operatorname{sgn}(\mathbf{w})$. Thus $T_{2}=\alpha(\pi)$. We also have FlatDif $=n-2 k$.

Case 3. w has a trailing string of 0 's but no leading string of 0 's. Again, FlatDif $=n-2 k$. Let $f$ be the location of the last 1 . Since $k$ is odd, $\operatorname{sgn}\left(\mathbf{w}^{f}\right)=\operatorname{sgn}(\mathbf{w})$. Thus, $T_{2}=\alpha(\pi)$.

Case 4. w has a trailing string of 0 's and a leading string of 0 's. Then both $\mathbf{w}^{s}$ from Case 2 and $\mathbf{w}^{f}$ from Case 3 arise. Thus, $T_{2}=$ $2 \alpha(\pi)$. Furthermore, FlatDif $=n-2 k-1$.

Equation (1) follows in each of the four cases.

As an example of this construction, let $n=12$ and $k=5$. Let

$$
\pi=511031169841227 \text {. }
$$

Then

$$
\mathbf{w}=110100001010 .
$$

Then $\hat{\pi}=51342, \operatorname{sgn}(\mathbf{w})=-1$, and $\alpha(\pi)=-1$. Then $U(\pi)$ has four elements, one with $\alpha=+1$ and three with $\alpha=-1$. Furthermore, $V(\pi)$ has seven elements. These will have corresponding $\mathbf{w}^{i}$ 's:

$$
\begin{aligned}
& 101100001010 \text { sgn }=+1 \text {, } \\
& 111000001010 \text { sgn }=-1 \text {, } \\
& 110010001010 \text { sgn }=-1 \text {, } \\
& 110100010010 \mathrm{sgn}=+1 \text {, } \\
& 110100000110 \text { sgn }=+1 \text {, } \\
& 110100001100 \text { sgn }=-1 \text {, } \\
& 110100001001 \text { sgn }=-1 \text {. }
\end{aligned}
$$

The first six of these pair off. The last has the same sign as $\mathbf{w}$. Thus, the left-hand side of (1) sums to -3 , which is $-1(n-2 k+1)$.

Theorem 6.1 has some interesting special cases. 
Corollary 6.2. For $n \equiv 1 \bmod 4,0 \in \operatorname{Spec}(T)$.

Corollary 6.3. For $n$ even, $-(n-1),-(n-3), \ldots,-1,1,3, \ldots$, $n-1 \in \operatorname{Spec}(T)$. For $n$ odd, $-(n-9),-(n-5), \ldots, n-5, n-9 \in$ $\operatorname{Spec}(T)$.

Because of the special form of the eigenvectors computed in Theorem 6.1 , we can produce an interesting vertex cover of $G_{n}$, although not a code. Call a set $C$ of vertices of $G_{n}$ a $k$-cover-code if every vertex of $G_{n}$ is contained in exactly $k$ of the closed neighborhoods of $C$.

COROLlary 6.4. If $n$ is even then $G_{n}$ has an $n / 2$-cover-code.

Proof. If $n$ is even then using the construction of Theorem 6.1 one can produce an eigenvector $\alpha$ with eigenvalue -1 all of whose entries are \pm 1 . If we let

$$
C_{\alpha}=\sum_{\sigma \in S_{n}} \alpha(\sigma) \sigma
$$

then $C_{\alpha} T_{1}=0$ by Proposition 3.1. The sum

$$
C=\frac{1}{2}\left(\widehat{S}_{n}+C_{\alpha}\right)
$$

has only coefficients of 1 and 0 and

$$
C T_{1}=\frac{n}{2} \widehat{S}_{n}
$$

so the permutations in the support of $C$ form an $n / 2$-cover-code.

7. Radon transforms. In this section we define a Radon transform on the group $S_{n}$ and discuss its invertibility. A similar question has previously been studied by Diaconis and Graham [DG]; however their definition is based on using all transpositions of $S_{n}$, while ours uses only the adjacent transpositions. As noted before, working with adjacent transpositions, which do not form a conjugacy class, presents different technical problems.

Let $\mathscr{F}$ be the set of functions from $S_{n}$ to $\mathbb{C}$. We define two transforms on $\mathscr{F}:$ For $f \in \mathscr{F}$ and all $\sigma \in S_{n}$ let

$$
R_{0}[f](\sigma)=\sum_{\{\tau \sim \sigma\}} f(\tau)
$$

and

$$
R_{1}[f](\sigma)=R_{0}[f](\sigma)+f(\sigma) .
$$

Observe that if we consider $\mathscr{F}$ to be a formal vector space generated by $S_{n}$, then the transforms $R_{0}$ and $R_{1}$ can be considered the linear 
transformations given by the matrices $A$ and $A+I$ respectively. The following theorem is then immediate from Theorem 2.7:

Theorem 7.1 (Compare with [DG, Section 4, Theorem 5]). For $n \geq$ 2 the transform $R_{1}$ is never invertible.

Unfortunately, we cannot be as conclusive in the case of the transform $R_{0}$ :

TheOREM 7.2 (Compare with [DG, Section 4, Corollary 2]). If $n \equiv$ $1 \bmod 4$, or $n=6,10,12$ then $R_{0}$ is not invertible.

Proof. The case $n \equiv 1 \bmod 4$ follows from Corollary 6.2. The other cases were checked by computer. See the table below.

8. Conjectures and additional remarks. We have compiled a table of the sets $\operatorname{Spec}\left(T^{\lambda}\right)$ when $\lambda$ is a hook. We give a portion of this table below. A $\checkmark$ indicates that the number is in $\operatorname{Spec}\left(T^{\lambda}\right)$, a $\bullet$ indicates that it is not, and a ? means that we do not know. When $\lambda$ is not a hook, we have found only four non-empty $\operatorname{Spec}\left(T^{\lambda}\right)$ : when $\lambda=3^{2}$, $2 \in \operatorname{Spec}\left(T^{\lambda}\right)$; when $\lambda=431,1 \in \operatorname{Spec}\left(T^{\lambda}\right)$; and the conjugates of these shapes.

Conjecture 8.1. $k \in \operatorname{Spec}(\widehat{T})$ implies $k \in \operatorname{Spec}\left(T^{\lambda}\right)$ for some hook shape $\lambda$.

Using the connection established by Theorem 4.4, our data seem to indicate that the only integer eigenvalues of $Y_{a, b}$ are in the set $\{-1,0,+1\}$.

CONJECTURE 8.2. The only possible integer eigenvalues of $Y_{a, b}$ are $-1,0,+1$.

Note that Conjecture 8.2 will not be a consequence of some bound on the largest eigenvalue. For $Y_{22}$, the eigenvalues include $\sqrt{5}$.

Powers of 2 and primes seem to have special patterns.

Conjecture 8.3. If $n=2^{l}$, then

$$
\operatorname{Spec}(\widehat{T})=\{ \pm(2 k-1) \mid 1 \leq k \leq n / 2\} \text {. }
$$

CONJECTURE 8.4. If $n$ is an odd prime, then

$$
\operatorname{Spec}(\widehat{T})=\{ \pm 1\} \cup\{n-4 k-1 \mid 0 \leq k \leq(n-1) / 2\} .
$$

We have already shown (Corollary 6.2) that $0 \in \operatorname{Spec}(\widehat{T})$ when $n \equiv 1 \bmod 4$. 
Table of $\operatorname{Spec}\left(T^{\lambda}\right)$ for $\lambda=n-k, 1^{k}$

\begin{tabular}{|c|c|c|c|c|c|c|c|c|c|c|c|c|c|}
\hline$n$ & $n-k$ & $k$ & o & 1 & 2 & 3 & 4 & 5 & 6 & 7 & 8 & 9 & 10 \\
\hline 2 & 2 & 0 & • & $\checkmark$ & & & & & & & & & \\
\hline \multirow[t]{2}{*}{3} & 3 & 0 & • & • & $\checkmark$ & & & & & & & & \\
\hline & 2 & 1 & • & $\checkmark$ & • & & & & & & & & \\
\hline \multirow[t]{2}{*}{4} & 4 & 0 & • & • & • & $\checkmark$ & & & & & & & \\
\hline & 3 & 1 & • & $\checkmark$ & - & - & & & & & & & \\
\hline \multirow[t]{3}{*}{5} & 5 & 0 & • & • & • & • & $\checkmark$ & & & & & & \\
\hline & 4 & 1 & • & • & • & • & • & & & & & & \\
\hline & 3 & 2 & $r$ & $r$ & • & • & • & & & & & & \\
\hline \multirow[t]{3}{*}{6} & 6 & o & • & • & • & • & • & $\checkmark$ & & & & & \\
\hline & 5 & 1 & • & • & $r$ & $r$ & $\checkmark$ & • & & & & & \\
\hline & 4 & 2 & $\checkmark$ & $r$ & $r$ & • & $\bullet$ & $\bullet$ & & & & & \\
\hline \multirow[t]{4}{*}{7} & 7 & o & • & - & • & • & • & • & $r$ & & & & \\
\hline & 6 & 1 & • & • & • & • & • & • & • & & & & \\
\hline & 5 & 2 & • & • & $\checkmark$ & • & • & • & • & & & & \\
\hline & 4 & 3 & • & $\checkmark$ & • & • & • & • & • & & & & \\
\hline \multirow[t]{4}{*}{8} & 8 & o & • & • & • & • & • & • & • & $\checkmark$ & & & \\
\hline & 7 & 1 & • & • & • & • & - & $\checkmark$ & • & • & & & \\
\hline & 6 & 2 & • & • & • & $r$ & • & • & • & • & & & \\
\hline & 5 & 3 & • & $\checkmark$ & • & • & $\bullet$ & $\bullet$ & • & • & & & \\
\hline \multirow[t]{4}{*}{9} & 9 & 0 & • & • & • & • & - & • & • & • & $\checkmark$ & & \\
\hline & 8 & 1 & • & • & • & • & • & $r$ & • & $\checkmark$ & • & & \\
\hline & 7 & 2 & • & • & $\bullet$ & • & $\checkmark$ & • & • & $\bullet$ & • & & \\
\hline & $\begin{array}{l}6 \\
5\end{array}$ & $\begin{array}{l}3 \\
4\end{array}$ & is & $\stackrel{r}{r}$ & $\stackrel{r}{\circ}$ & $\checkmark$ & $\bullet$ & : & $\bullet$ & $\bullet$ & : & & \\
\hline \multirow{5}{*}{10} & 10 & 0 & . & & . & . & 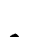 & 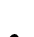 & 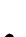 & & & & \\
\hline & 9 & 1 & $\vdots$ & $\vdots$ & : & $\dot{\bullet}$ & : & • & $\dot{\bullet}$ & $\dot{s}$ & : & ? & \\
\hline & 8 & 2 & • & • & 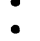 & . & $\checkmark$ & $\checkmark$ & $i$ & ? & 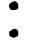 & $\dot{\bullet}$ & \\
\hline & 7 & 3 & • & • & $r$ & $\checkmark$ & $\checkmark$ & • & • & • & - & - & \\
\hline & 6 & 4 & $\checkmark$ & $r$ & $r$ & • & - & • & • & • & - & - & \\
\hline \multirow[t]{6}{*}{11} & 11 & 0 & • & • & • & - & • & • & - & - & - & • & $\checkmark$ \\
\hline & 10 & 1 & • & • & • & • & • & • & • & • & • & • & • \\
\hline & 9 & 2 & • & • & • & • & • & • & $\checkmark$ & • & • & • & • \\
\hline & 8 & 3 & • & • & • & • & • & • & - & • & • & • & • \\
\hline & 7 & 4 & • & • & $\checkmark$ & • & • & • & • & • & • & • & • \\
\hline & 6 & 5 & - & $\checkmark$ & • & • & • & $\bullet$ & • & • & • & • & • \\
\hline \multirow[t]{6}{*}{12} & 12 & 0 & • & • & • & • & • & • & • & • & • & • & • \\
\hline & 11 & 1 & • & • & • & • & • & • & • & • & $\checkmark$ & $\checkmark$ & $\checkmark$ \\
\hline & 10 & 2 & • & • & • & - & • & • & $r$ & $r$ & $\checkmark$ & • & • \\
\hline & 9 & 3 & • & • & • & • & $\checkmark$ & $\checkmark$ & $r$ & • & • & • & • \\
\hline & 8 & 4 & • & • & $\checkmark$ & $r$ & $\checkmark$ & $?$ & $?$ & $?$ & $?$ & $?$ & $?$ \\
\hline & 7 & 5 & $?$ & $\checkmark$ & $?$ & $?$ & $?$ & $?$ & ? & $?$ & $?$ & $?$ & $?$ \\
\hline
\end{tabular}

Conjecture 8.5. If $n \equiv 0 \bmod 6$, then $0 \in \operatorname{Spec}(\widehat{T})$.

More generally,

Conjecture 8.6. If $n \equiv 0 \bmod 6$, then

$$
\operatorname{Spec}(\widehat{T})=\{-(n-1),-(n-2), \ldots,-1,0,1, \ldots, n-1\} \text {. }
$$


Acknowledgments. We would like to thank R. Stanley for his many helpful remarks during the course of this research.

\section{REFERENCES}

[Ba] L. Babai, The spectra of Cayley graphs, J. Combinatorial Theory Ser. B, 27 (1979), 180-189.

[Be] C. Berge, Principles of Combinatorics, Academic Press, New York, 1971.

[Bj] A. Björner, Orderings of Coxeter groups, Proc. AMS-NSF Conference on Combinatorics and Algebra, Boulder 1983 (ed. C. Greene), Contemporary Mathematics, 34 (1984), 175-195.

[DG] P. Diaconis and R. Graham, The Radon transform on $Z_{2}^{k}$, Pacific J. Math., 118 (1985), 323-345.

[DS] P. Diaconis and M. Shahshahani, Generating a random permutation with random transpositions, Z. Wahrsch. Verw. Gebiete, 57 (1981), 159-179.

[CDS] D. M. Cvetkovic, M. Doob and H. Sachs, Spectra of Graphs, Theory and Application, Academic Press, New York, 1980.

[GM] A. M. Garsia nd T. J. McLarnan, Relations between Young's natural and the Kazhdan-Lusztig representations of $S_{n}$, Adv. in Math., 69 (1988), 32-92.

[KL] D. Kazhdan and G. Lusztig, Representations of Coxeter groups and Hecke algebra, Invent. Math., 53 (1979), 165-184.

[Kn] D. E. Knuth, The Art of Computer Programming, volume 3, Addison-Wesley Publishing Co., Reading, MA, 1973.

[JK] G. D. James and A. Kerber, The Representation Theory of the Symmetric Group, Addison-Wesley, Reading, Mass., 1981.

[RT] O. Rothaus and J. G. Thompson, A combinatorial problem in the symmetric group, Pacific J. Math., 18 (1966), 175-178.

[SW] D. W. Stanton and D. E. White, Constructive Combinatorics, Springer-Verlag, New York, 1986.

Received May 23, 1988 and in revised form September 19, 1988. This work was partially supported by NSF grants DMS-8612466, DMS-8700995 and DMS-8501332.

UNIVERSITY OF MINNESOTA

MiNNEAPOLIS, MN 55455 



\section{PACIFIC JOURNAL OF MATHEMATICS EDITORS}

\author{
V. S. VARADARAJAN \\ (Managing Editor) \\ University of California \\ Los Angeles, CA 90024-1555-05 \\ Herbert Clemens \\ University of Utah \\ Salt Lake City, UT 84112 \\ Thomas ENRIGHT \\ University of California, San Diego \\ La Jolla, CA 92093
}

R. FINN

Stanford University

Stanford, CA 94305

Hermann FlaschKa

University of Arizona

Tucson, AZ 85721

VAughan F. R. Jones

University of California

Berkeley, CA 94720

Steven Kerckhoff

Stanford University

Stanford, CA 94305
RobION KIRBY

University of California

Berkeley, CA 94720

C. C. MOore

University of California

Berkeley, CA 94720

HAROLd STARK

University of California, San Diego La Jolla, CA 92093

\begin{tabular}{|c|c|c|c|c|}
\hline & ASSOCIA & E EDI & & \\
\hline R. ARENS & $\begin{array}{l}\text { E. F. BECKENBACH } \\
(1906-1982)\end{array}$ & NeumanN & $\begin{array}{c}\text { F. WoLF } \\
(1904-1989)\end{array}$ & K. Yoshida \\
\hline & SUPPORTINC & INSTI & TIONS & \\
\hline UNIVERSITY & ARIZONA & UNIVERS & OF OREGON & \\
\hline UNIVERSITY & BRITISH COLUMBIA & UNIVER & OF SOUTHEI & CALIFORNIA \\
\hline CALIFORNIA & STITUTE OF TECHNOLOGY & STANFOI & UNIVERSITY & \\
\hline UNIVERSITY & CALIFORNIA & UNIVER & OF HAWAII & \\
\hline MONTANA S & TE UNIVERSITY & UNIVER & OF TOKYO & \\
\hline UNIVERSITY & NEVADA, RENO & UNIVER & OF UTAH & \\
\hline NEW MEXIC & TATE UNIVERSITY & WA & N STATE UN & ERSITY \\
\hline OREGON ST & UNIVERSITY & & OF WASHINC & $\mathrm{ON}$ \\
\hline
\end{tabular}




\section{Pacific Journal of Mathematics}

\section{Vol. 143, No. $1 \quad$ March, 1990}

Walter Bergweiler, On the fix-points of composite functions $\ldots \ldots \ldots \ldots \ldots 1$ Aldo Biancofiore, Maria Lucia Fania and Antonio Lanteri, Polarized surfaces with hyperelliptic sections $\ldots \ldots \ldots \ldots \ldots \ldots \ldots \ldots \ldots$

Ciprian Borcea, Deforming varieties of $k$-planes of projective complete

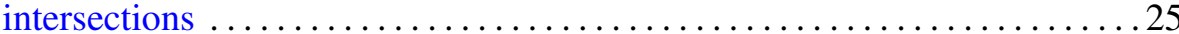

Morton Brown, Fixed points for orientation preserving homeomorphisms of

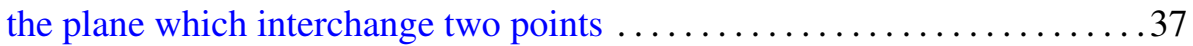

Hao Zhi Chuan, Note on the inequality of the arithmetic and geometric means

Paul Henry Edelman and Dennis E. White, Codes, transforms and the

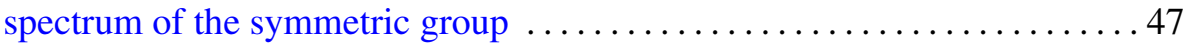

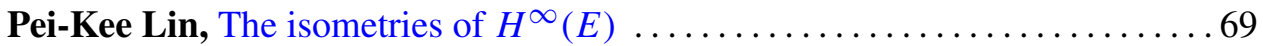

James J. Moloney, Residue class domains of the ring of convergent

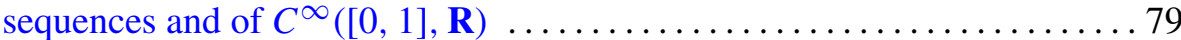

Zhong-Jin Ruan, The structure of pure completely bounded and completely

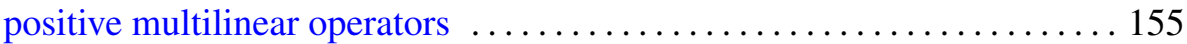

Wolfgang Ruess and William H. Summers, Weakly almost periodic semigroups of operators

Gideon Schwarz, A pretender to the title "canonical Moebius strip" . . . . . . 195

Ryszard Szwarc, Banach algebras associated with spherical representations of the free group 